\title{
ALED System to Provide Mobile IoT Assistance for Elderly and Disabled
}

\author{
${ }^{1}$ Nikhil Chaudhari ${ }^{*}{ }^{2}$ Akash Gupta and ${ }^{3}$ SSV Raju \\ School of Computer Engineering (SCOPE), \\ Vellore Institute of Technology (VIT) University, Vellore, \\ 632014 Tamil Nadu, India \\ Inikhil.chaudhari2013@vit.ac.in, ${ }^{2}$ akash_gupta.2013@vit.ac.in, \\ srikanta2192@gmail.com
}

\begin{abstract}
The United Nations estimates that about 15 percent of the tyorld's population lives with some type of disability. Equally important is the increasing life expectangy rate of elderly people. People falling in any of these categories are great difficulty in living an independent life and have to be dependent on others. Interner-finings (IoT) presents immense potential in solving these problems. IoT is a concept by which everyday objects can communicate with each other via a network. This enables us to collect and store the data from these devices in the Cloud. Although there are existing systems that help to solve some of these problems; however, a comprehensive system is missing. In this paper, we propose a unified system ALED(Assisted Civing for the Elderly and Disabled) using Google Brillo and Weave platform to solve both of these problems. The system provides a solution by collecting data from $10 T$ deviceS (t.g., smart sensors, wearable devices, tags) and utilizes the Cloud to process the information from database. The system enables the elderly and disabled to Tave their life independently and at the same time make it more pleasing.
\end{abstract}

Keywords Intermet of Things (IoT), network, Cloud, ALED, Brillo, Weave, sensor, wearable, database.

\section{Introduction}

The increasing population of people with disabilities and the elderly presents a big challenge in the areas of home assistance, outdoor aid, self-assistance, healthcare and emergencerservices. With old age comes many health related problem, which hinder their daily actiyities. Some examples of such problems are limited motor functionality due to arthritis, health related issues like high blood, diabetes, heart ailments and other general healtb related issues. These problems can even include memory loss, poor vision, reduced hearing capacity. People with disabilities have to undergo hardship throughout their life. Majority of them suffer from one or more disabilities of vision, hearing, speech and physical disability. As such they have a hard time to live their life in a stress-free manner.

With the advancements in ubiquitous computing, communication and cloud technologies, a whole new set of universal systems can be developed that can solve the problems mentioned above in real-time. Internet of Things (IoT), enables all devices to communicate to each other in real-time. These devices can range from a simple light bulb, a normal shoe to sophisticated monitoring cameras and even aircrafts. With the widespread adoption of smart-phones the concept of IoT can be easily realized. All the

Nikhil Chaudhari is the corresponding author. 
connected devices generate enormous amounts of data. Cloud technologies can utilize this data by processing it and provide information. This information can be utilized by connected devices to provide better services. Thus, by using IoT we can make the objects in our environment a part of internet. Hence IoT paves a way for the near future where everything related our lives will be integrated system.

This paper aims to solve the problems faced by disabled and elderly by proposing ALED (Assisted Living for Elderly and Disabled) which leverages IoT. The proposal is a modular system which can cater to needs of both disabled and elderly. We try to achieve this by maximizing the use of smart phone, converting the regular devices into "smart devices" and utilizing the network and cloud services extensively. ALED system will use Google Brillo [8] which is an OS (Operating System) specifically designed to enable hardware to be ready for IoT. Weave [10] is a communication platform, which (enables these Brillo enabled hardware to communicate with one another and also other snart devices which do not run on Brillo. Brillo combined with Weave provides a great opportunity to standardize IoT by providing a common OS and standard communication platform. With Brillo and Weave it becomes very easy to make the connectivity with Android and iOS because of the availability of native APIs. The ALED system comprises of three modules namely: OBM (On-Body Modułe) HAM (House Assistance Module) and OAM (Outdoor Assistance Module). All these three modules ane themselves modular in structure and versatile in functionality. This means some components in every module can be swapped as per the need of user. This in-turn.sares a lot of cost and make the system more specific for a disabled person or elderly. The OBM houses a bunch of sensors that need to be attached to the ursers at all imes. These sensors include a Body Vital Sensor (BVM), a RFID (Radio Frequeney Identification) reader and text-to-speech module, etc. The HAM is a web of subsystems which are installed inside the home environment of the user. HAM includes electronically controlled home systems like switches, appliances, latches, locks, windows. A very important feature provided by HAM is the identification of electronic and non-electronic items in the home environment. This is achieved by embedding all concerned objects with native RFID tags which can then beread by an RFID reader. The HAM also includes a facial recognition based guest monitoring system which utilizes a cloud database to inform the user about the identity of visitor. The QAM consists of smart accessories that will be useful when the user goes outside his home environment and also to some extent inside the home environment. The agcessories include: smart shoe, smart glove, smart cane, an electronically controlled wheelchair, etc.

The proposed ALED system can cater to needs of both elderly and disabled by providing assistance in the areas of healthcare, general daily activities, emergency assistance and assistance while travelling. The system tries to integrate maximum amount of dail objects in the cloud by connecting them to the Internet. This is done by the use of RFIDs and connecting the sensors to the cloud using Wi-Fi and Bluetooth LE(Low Energy).The ALED system can be realized today using a standard IoT platform such as Brillo and Weave, along with the necessary sensors and actuators.

\section{Related Works}

IoT is a field which combines various disciplines of Computer Science and Technology such as embedded systems, computer networks, cloud technologies and operating systems. It is a relatively new concept which has been made possible by the advancements in disciplines mentioned above. Thus, available quantity of works in this field is relatively low compared to other works in ICT (Information and Communication Technologies).

Mari Carmen Domingo has proposed a system that provides assistance to people with disabilities [1]. The system utilizes currently available sensors along with advanced micro and nano sensors. This system does not differentiate between the challenges faced by the 
disabled in the home environment and outdoor environment. Also some of the bio and nano sensors such as retinal implant, brain computer interface cannot be realized at the consumer level. A real-time system of the management of Parkinson's disease using IoT and wearable technologies has been proposed [2]. The paper discusses about management of health issues with focus on Parkinson's disease as a representative model. They propose to a threefold approach: they mentioned about the importance of IoT in health care, combining wearable technologies with IoT and engagement of patients in the treatment process. I. F. Akyildiz. et al., [3]introduce the internet of Bio-Nano things (IoBNT). They discuss about sensors which monitor biological activities at the cellular level and intrabody sensing and actuation networks, and environmental control of toxic agents and pollution. The bio sensors mentioned in the study operate at nano scale by combining nano-technology and synthetic technology. Such sensors are currently not available at the consumer level and their feasibility is still being evaluated.

A Smart cane has been developed for the visually impaired people by Edoardo D'Atri, Carlo Maria Medaglia and Alexandru Serbanati [4]. They also mention about the shortcoming related to design of cane and lack of responsineness of RFID passive tags. Faster movements by the user may miss the tags and loss of signals, thus leading to wrong path guidance. Thus more developmental work is needed to make the RFID tagging process much more reliable. A similar Navigation System for the blind using a smart cane for Indoor Environments [5] has been proposed by Sakmongkon Chumkamon. The system takes considerable time to establish connection and is somewhat large for convenient daily usage. The system also needs to improve on its text-to-speech functionality. Our system aims to use the already funished technology of Android API's for text-to-speech and use newer sensors and micro-controllers which have reduced size in comparison to the above system. Another version of RFID and GPS Integrated Navigation System for the Visually Impaired [6] is proposed by Kumar Yelamarthi which uses GPS

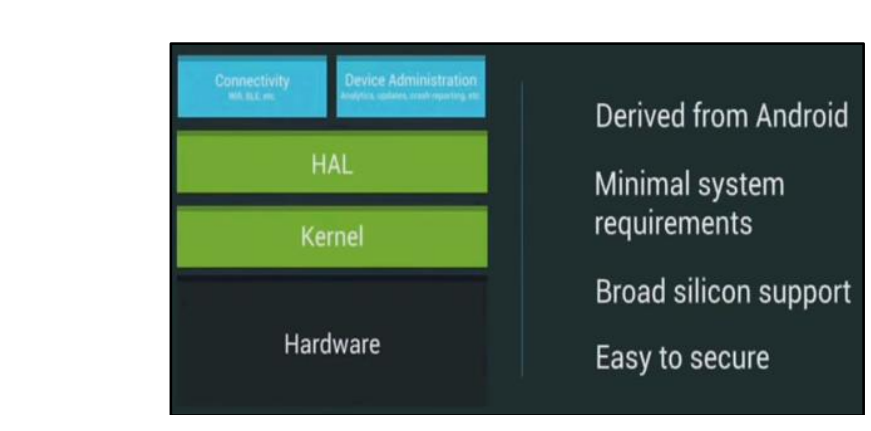

Figure 1. Ah Overview of the Architecture of Google Brillo (Source: Google)

for gaiding the blind. This system uses vibration motors for providing instructions directly to the user thereby increasing accuracy in noisy environments but uses an external robot as a guide for the visually impaired person which does not provide a wide coverage as it is easily obstructed by stairs, puddles, snow and other off-road environments. The main objective of this system is to provide guidance to the blind user in outdoor environment where all these obstacles are a common sight. 


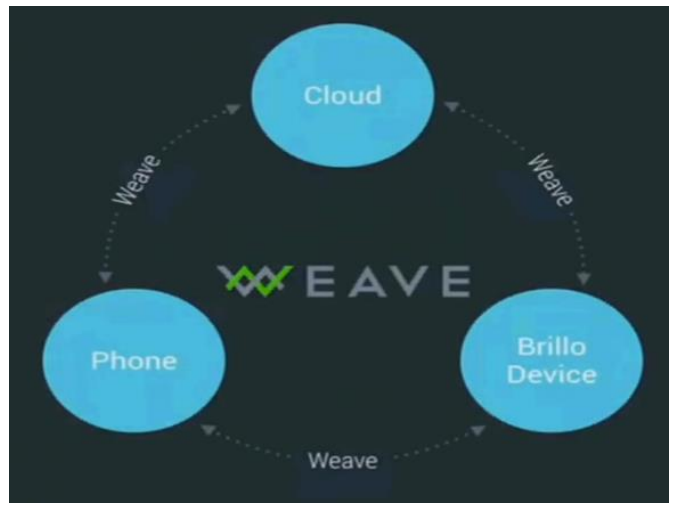

\section{Figure 2. Connectivity Options provided by Google Weave (Source: Google)}

A good gesture controlled system [7] has been proposed by Suat Akyo/. This/system is focuses on controlling multimedia operation inside automobiles. This system can also be used in the home environment for people with limited mobility. The system uses Near Infrared light for lighting the gesture area and modified CCD (Charged Coupled Devices) camera. We can also use 3 CCD camera which will help in better image acquisition finally leading to better results. Also dynamic gestures might help in increasing system's intuitiveness and naturalness. Thus we can conclyde that there does not exist complete all in one system which provides assistance in health-cate as well as home-automation, navigation etc.

\subsection{Challenges}

The main factor which would limit the functionality of the ALED system would be reliable connectivity to the Internet. Developing countries such as India have yet to see widespread adoption of reliable connectivity technologies such as Wi-Fi, 3G/4G. These facilities are only ataitable to a handful of people currently. Thus it is necessary that these technologies see greater adoption rate for the success of IoT platforms. The ALED system is comprehensive and thus there is a curve involved to operate such systems. It is essential that the user Is willing to overcome the learning curve to maximize the potential benefits of the system.

Currently, Brillo anc Weave are under heavy development and the platform is still accessible using invite. Hence there are currently there few commercially available smart devices that are compatible with Weave. But it is expected that once the platform is opened for public use it would see widespread adoption. Moreover, it would lead to certification of the exiting smart devices to work with Weave. The open source nature of this platform would be the major driving force behind its adoption rate. It is also worth mentioning that a robust cloud infrastructure is essential for the realization of any IoT system.

\section{Proposed System Architecture}

In this section we discuss in-depth about the architecture and functionality of the proposed system. We first begin by discussing about the benefits of using Google Brillo and Weave as standards for IoT. Then we discuss in detail about all the three modules.

\subsection{Google Brillo}

Google introduced Project Brillo [8] at the Google I/O 2015 as a underlying standard OS to power all IoT devices. Brillo is derived from Android OS. It is a modified to be used with devices with low-power and memory constrained IoT devices. It retains the functionalities of lower layer of Android namely the HAL(Hardware Abstraction Layer) 
and Android Kernel. Since it based on Android it supports Wi-Fi and Bluetooth-LE (Bluetooth Low Energy) communication standards. Devices which don't have built-in Wi-Fi module/Mobile Data functionality are connected to the cloud through the user's smart-phone using Bluetooth-LE. Brillo supports Intel x86, ARM and MIPS instruction sets [8]. It has built in security features and supports OS updates directly from Google. Brillo is currently supported on Intel Edison, Qualcomm Dragon-board 410c, Andromeda Box Edge and Creator CI20. All these boards have integrated Wi-Fi and Bluetooth modules. They all have capable SoC (System on Chip) with plenty of processing power, RAM and storage.

Currently there is no standard OS and communication platform for IoT devices. Existing platforms such as NEST and Apple HomeKit are primarily focused towards home automation. Both these platforms already automated some home devices like thermostats, light-bulbs and smoke detectors. There are other manufacturers also whoalso produce smart devices like Phillips, Xiaomi, Whirlpool, etc.

All these platforms have their own proprietary OS and communications platforms. This means that smart devices running on one platform cannot communicate with devices running on other. This leads to heavy fragmentation among the IoT devices. This also discourages medium and small manufacturers to develop smart devides as they do not have access to a polished OS and communication platform specifically tailored for IoT. Google acquired Nest 2014, which was one of the first pioneers in home automation field.

Developers from NEST, Android and the Chrome OS teams have collaborated to develop Brillo and Google Weave as a standard for IoT. Britho aims to solve this problem of fragmentation of IoT platforms by proyiding a fundamentally new approach to IoT by developing an end-to-end solution Nest and Apple HomeKit focus mainly on home automation whereas Brillo is a Tersatle OS that is applicable to all fields where ever the concept of IoT can be realized such as healtit-care, agriculture, transportation, etc. Thus it is not limited to the home automation sector unlike the currently available platforms. Brillo simplifies software development for IOT with an embedded OS, core services, developer kit, and developer console. Brillo allows us to choose our hardware so the system can have different boards based on the price. Brillo provides easy move from prototype to production.

Although Nest/s Weave is proprietary it shares the same goals, schemas and data model with Google's Weave. So they will be compatible while remaining distinct. This ensures that products built oneither platform will be able to work with one another. This would be very helpful as there are already manufacturers such as Phillips, Whirlpool, Amazon, Chamberlain and many others which develop a wide variety of products which are compatible with Nest.

Currenty, Brillo and Weave are under heavy development and are available for use on an invite basis. Both these platforms have tremendous potential to develop as the uniform platform. Further announcements are expected in 2016. Once this platform becomes publiely available it should see widespread adoption. The broad availability of Brillo devices will help to develop a universal and simplified IoT eco-system.

\subsection{Weave}

Weave [10] is communication's layer using which IoT devices can talk to each other. In simple word it is a common language for devices to talk with each other and to the cloud and also with smart-phones. It consists of standardized schemas and data model such as an "unlocked" and "locked" which represent the state of a lock. This helps all other devices in eco-system to understand the state of one another. In addition to the standard schemas provided by Weave, developers will have an option to submit their own custom schemas. This allows for a Weave Certification program so that anything that is Weave certified can work together. This means that smart devices which are already utilize platforms such as Apple HomeKit, Samsung SmartThings, Nest and other 
proprietary ecosystems only need to be Weave certified to operate in IoT system powered Brillo and Weave. This removes the need to develop new smart devices from scratch to be able to work with Weave, which in turn speed development process, saves cost and simplifies the IoT architecture.

Weave provides device setup, phone-to-device-to-cloud communication, and user interaction from mobile devices and the web. It is cross-platform and follows a modular approach. This means that systems can run Brillo and Weave together or use Weave with the existing system. Weave provides powerful developer API's (Application programming Interface) such Voice API to remotely control devices using voice.

Another major factor why the proposed system utilizes Brillo and Weave is because they provide a consistent user experience. This is possible as Brillo is derived from Android and thus any android device (majority of them being smart-phones) would easily recognize a device running Brillo or Weave and the users would be presented with the standard android device setup interface. The application development ecosystem for Brillo and Weave is also very similar to that of Android and thus the learning curve is minimized. All this makes it easy for developers to build elegant apolications for Brillo devices and applications that would control these Brillo and Weave enabled devices.

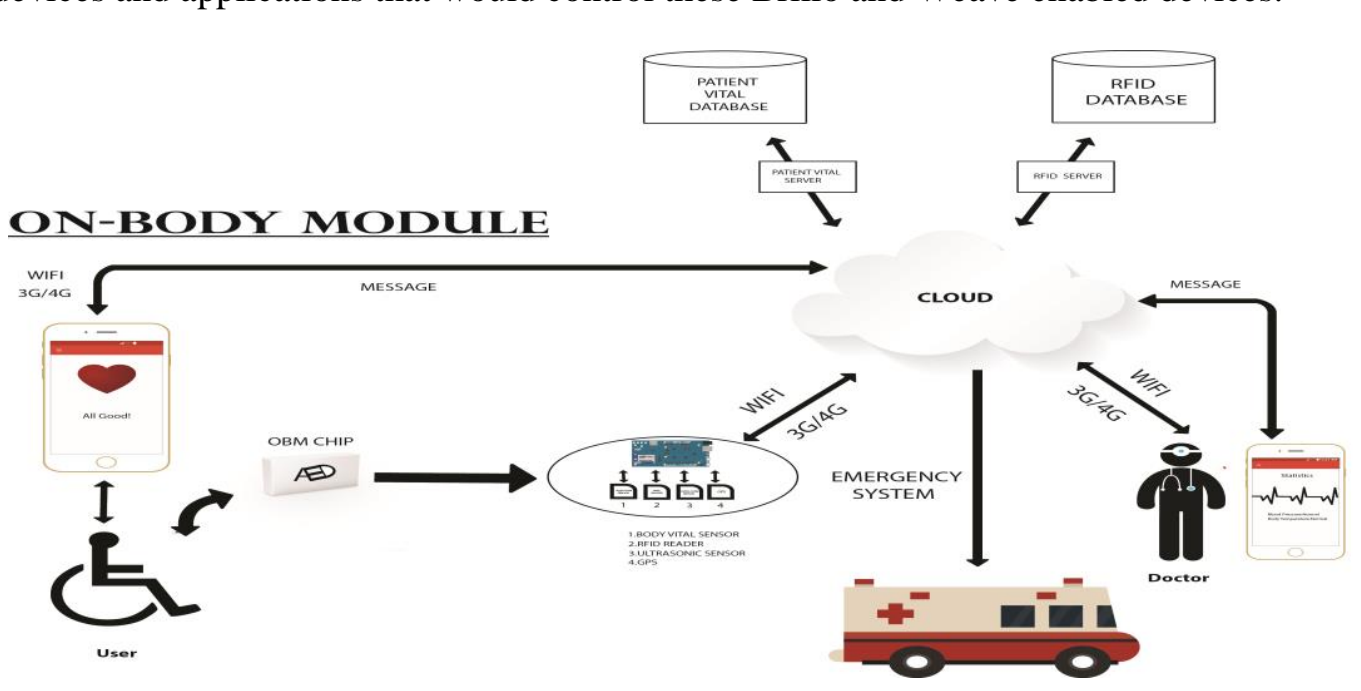

Figure 3. Architectural Representation of the On-Body Module

\subsection{OBM (On-Body Module)}

This module is powered by a Brillo compatible board like Intel Edison or Qualcomm's Dragon-board 410c. The system would have a BVM (Body Vital Sensor), a RFID reader, a GPS Module (Global Positioning System) and an ultrasonic sensor. The system is complemented by using a smart-phone as it adds much additional functionality that would highly useful for the users. The proposed architecture is shown in Figure 3.

3.3.1 BVM (Body Vital Sensor): The BVM houses sensors that monitor the heart rate, blood pressure, body temperature, blood sugar levels and the respiratory rate. The BVM can be used at certain intervals or on an on demand basis. Except for the blood sugar monitor all the other vital signs can even be monitored continuously but that may not be required by the user. The vital signs read by the BVM and then send to the cloud. The data is stored in a Patient Vital Database (PVM) on a server in the cloud. This data can then be accessed by the doctor or the medical staff remotely using their smart-phone. After analyzing the data, the doctor can generate a health report for the user. The user is then informed about his health report on his smart-phone. This negates the need to go to 
the hospital for regular check-ups which will be helpful to both the elderly and the disabled people who find it difficult to visit medical facilities frequently.

In addition to this on-demand monitoring of patient vitals by the medical staff there is also an Emergency Response System that would monitor the PVM at all times. Whenever the combination of some vital signs fall below a certain safe level (these levels would be specified by medical experts) the emergency response software running on a server in the cloud would automatically alert the medical authorities and initiate an ambulance request. Due to added GPS module inside the OBM chip this system would be highly effective even when the user is outside his house. The GPS would assist the medical authorities to pin-point the exact location of the user to deliver fast and efficient medical response.

3.3.2 RFID Reader: The OBM also houses an RFID (radio-frequency identification) reader. This meant to assist the blind people to identify the objects in their surfourdings. In the HAM we propose to embedded RFID tags in all objects inside the houtse and even in some object outside on the streets. The RFID reader transmits the id-string to the cloud server which returns the object information back. This information can be then displayed on the user's smart-phone. This feature is explained in detail when we discuss about the HAM.

3.3.3 Ultrasonic Sensor: The OBM has an integrated caltrasonic sensor for collision avoidance by detecting the obstacles in the user's path. Multiple ultrasonic sensors can be used to increase the accuracy. This functionality wills be of great help to the blind users. The ultrasonic sensor sends out sound from onesside and receives it back using the sensor on the other side. It measures the time intervalbetween sending the signal and receiving the echo to determine the distance to an object. Here the object can be an obstacle in the path of a blind user. If the ultrasonic sensordetects an object in close proximity it can alert the user using sound or even vibration for a deaf user. The user can then alter his walking speed and take necessary precautions to avoid collision.

3.3.4 Role of Smart-phone: The user's smart-phone plays a vital role to increase the effectiveness of the OBM Rather than including necessary actuators like speakers, vibrators and display we propose to utilize the built-in features of the smart-phone.

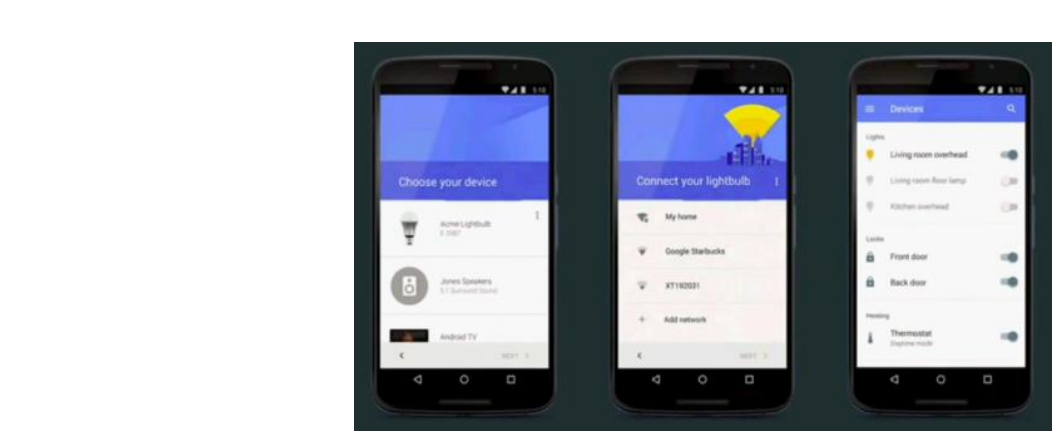

Figure 4. Demo of Android Application to Control Home Appliances like Bulb, Speakers, TV 


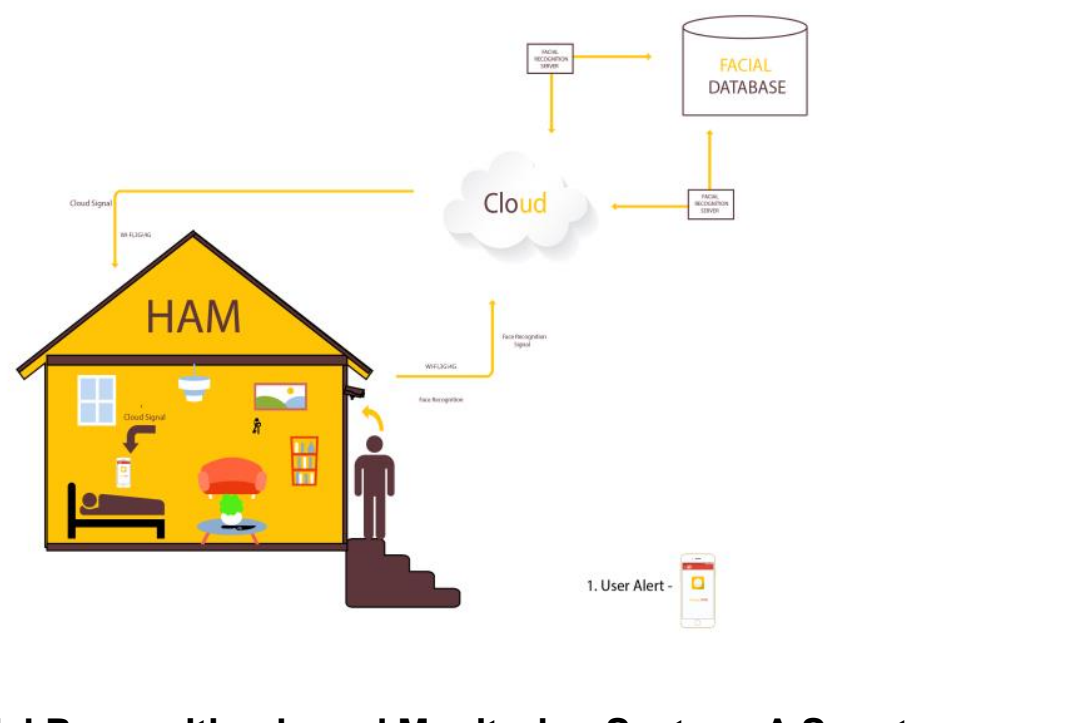

Figure 5. Facial Recognition based Monitoring System A Smart camera Captures the Visitors Facial Data and Transmits it to the Cloud where it is matched against a Facial Database. The Results are Delivered to User's Smart-Phone through Cloud

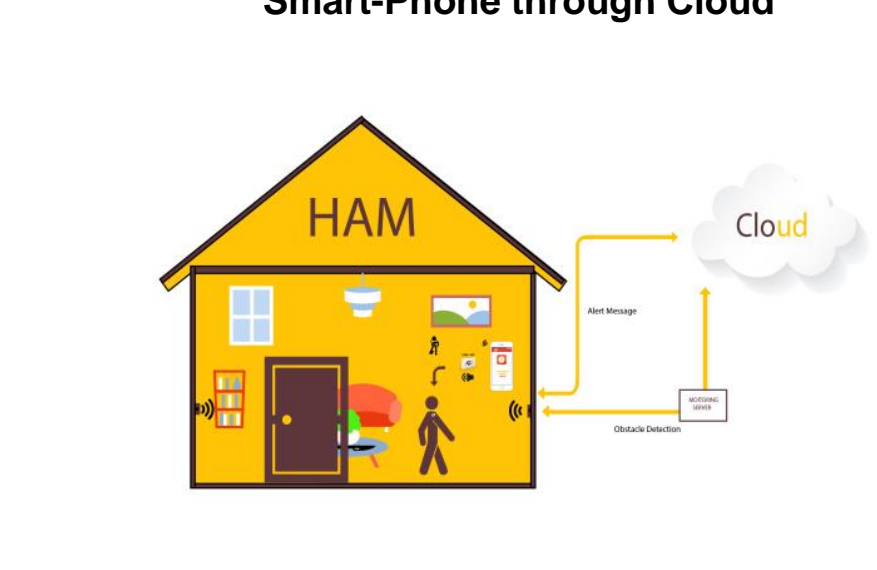

Figure 6. Collision Avoidance Inside the House using the Ultrasonic Sensors Mounted on the Walls

Including these functionalities inside the OBM chip would make it bulkier and at the same time provide redundant functionality. Android and iOS smart-phone have numerous as $1 \mathrm{~s}$ tive-features provided natively by the OS. Features such as Text-to-Speech would be use ful for blind users to interpret their health reports and other textual information. Smartphones even have applications that can scan physical documents using their inbuilt camera and create a digital copy. Then using the Text-to-Speech application these documents can be read out the deaf users. Due to advancements in Machine Learning and Voice Recognition it is now possible to provide reliable Speech-to-Text functionality. The smart-phone uses its microphone to collect the speech data. This data is then transmitted to the cloud servers which processes it and send back the textual information. This functionality is essential for the deaf users. Now it is even possible to control the functionality of the smart-phone and its applications by voice. Many such virtual assistance applications like Google Now (on Android) and Apple's Siri (on iOS) have become extremely popular.

Hence we recognize the importance of smart-phones and the various assistive features they offer. Thus we can utilize the user's smart-phone to provide these functionalities 
rather than including modules for them in the ALED system. This would save cost and at the same time provide a better experience to the user.

\subsection{HAM (House Assistance Module)}

This module consists of four major subsystems which provide different functionalities while the user is inside his home environment. These functionalities are facial recognition, household object identification, collision avoidance inside the house and control of lighting, fans, appliances etc. The user's smart-phone will serve as the tool to control the functionality and to receive information. These subsystems need not be powered by Brillo but should be Weave certified so that all the devices can communicate with each other and with the cloud.

3.4.1 Facial Recognition based Guest Monitoring System: The system usess a Weave certified wireless camera which is installed at the main entrance of the user's house. An example of a camera with such functionality can be the Nest Cam (it yses Nest's proprietary Weave). Whenever a visitor arrives at the entrunce the camera would take picture of the visitor's face. This facial data would then be sent to the cloyd where a facial recognition server would match the face data against the facial information already stored in the facial database. The results will then sent by the server directly to users the users smart-phone. The user can then unlock the door for an expected visitor or communicate with the visitor via the intercom installed at the main entranee using his smart-phone. The system will have option to save the face of $\mathrm{a}$ new guest. This will also help the user to alert the law enforcement agencies in case an offender arrives at the house. The law enforcement agencies could maintan facial database of all the criminals and then this database could be used by thecarnera installed at the house of the user.

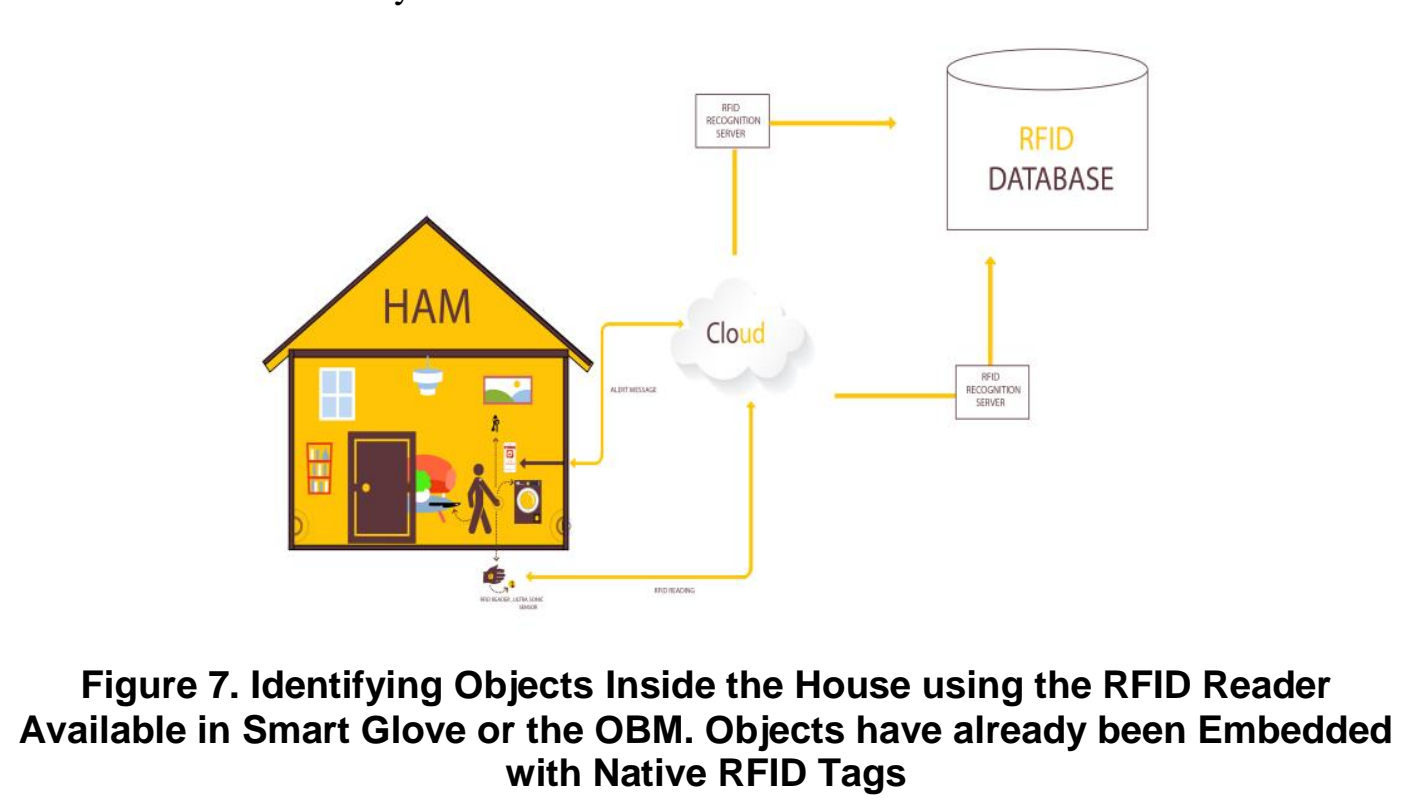




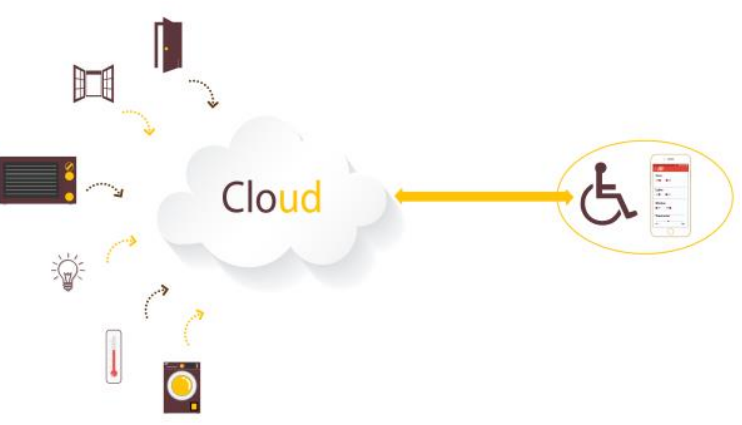

\section{Figure 8. Using the Smart-Phone to Control the Functionality of Various Electronic Devices inside the House such as Smart Blubs, Windows, Thermostats, Locks, etc.}

This system would be helpful to both the elderly people and disab ed people with limited motor functionality. Many elderly people suffer from arthritis and it is not feasible for them to frequently move around the house. The system dvill also be very useful for blind people who cannot recognize an unknown person or people who are restricted to wheel-chair. They do not need to get up from their beds to go to the door and can simply use their smart-phone to identify the visitor. This system will be equally useful to the general public to make their home more secure and maximize the use of IoT.

3.4.2 Controlling the Home Environment and Appliances: This subsystem is used to control the user's home environment involving the lighting, fans, doors, locks, windows, thermostat, car garage etc. It can also control appliances such as washing machine, oven, air-conditioner etc. There are two approaethes that can used to achieve this. The first approach is a pretty dated one and offers very less control over the functionality of these appliances. This approach involves controlling the power supply of these appliances and other electronic devices by connecting them to the microcontroller using necessary equipment. The microcontroller shbuld also have a Wi-Fi module. The user can then control the power supply using h)s smart-phone. But this approaches will only allow the user to turn them on or off and does not offer any control over their operation.

The second approach which we propose in the ALED system is the "smart connected devices approach". For this we need smart Weave certified devices and appliances. Such devices are expected to be available in the near future. But we already have many devices which already work 'with Nest's Weave [10] for example the Yale Linus which is a smart lock for Nest homes, Philips Hue Smart LED Light Bulbs, smart fans from Haiku, Whirlpoorsmart Washing Machine, Smart plugs from Zuli, Nuheat SIGNATURE Wi-Fi thermostaf and many more. We have already discussed that it is expected that devices which currently supported Nest's Weave should be compatible with Google's Weave. Other non Weave smart devices only need to undergo Weave certification to be compatible for use in the ALED system. They don't need to be redesigned from the ground up.

The user's smart-phone would be the point of access to control the functionality of these smart devices in the home environment. As all these devices are connected to the cloud they can even be controlled from outside the home environment making the experience even more seamless. This would remove the need to manually control them by moving through the different areas inside the house. This subsystem will be useful not only to the elderly and the disabled but also to the general user. All this would make it life easier for the elderly and the disabled by reducing their movements inside the house and be less dependent on others to perform such basic yet necessary tasks. 
3.4.3 Object Identification using Embedded RFID Tags: A major problem face by blind users is the lack of ability to identify the objects in their surroundings accurately. This problem is aggravated when the blind person is outside his house. In some cases, the objects in the surroundings can cause harm to the user for example sharp objects like knifes, electrical sockets. Our approach to solve this problem is to embedded all important object in the house with RFID tags containing information about them. We can also tag objects outside the house to indicate street names, landmark information, bus stops etc. This would help the blind users in commuting with ease outside their homes.

Objects like ovens, washing machine, keys, books, medicines etc. can be embedded with RFID tags. These tags can be read by using RFID readers embedded in the smart cane and smart glove. These two are discussed in detail in the OAM section. Even the RFID reader embedded in the OBM chip can be used to read to read the tags. The RFID string that is read is transmitted to the cloud using inbuilt Wi-Fi module/Mobile data or can be first transmitted to the user's smart-phone using Bluetooth-LE and thep to cloud from the smart-phone. The RFID server in the cloud would search for the string in the RFID database and send the results back to the user's smart-phone. The Government can also embed RFID tags in the streets and public places and maintan a public RFID database to assist the blind commuters.

3.4.4 Ultrasonic Sensor Mounted on the Walls: The walls in the house can be mounted with multiple ultrasonic sensors. These sensors would becconnected to the cloud. When the user comes in close proximity of the wall in the house the monitoring server in the cloud would automatically generate ancalent to the user's smart-phone. This functionality is targeted towards making movement inside the house easier for the blind users. The ultrasonic sensor mentioned here and the one in the OBM are alternatives for each other. But the embedded ultrasonic sensors in the walls inside the house would provide wide coverage and better collision detection in the house whereas the sensor in the OBM is general purpose one and can be used ourside.

\subsection{OAM (Outdoor Assistance Mddule)}

Providing assistance to elderly and disabled when they are outdoors is also an important aspect of the ALED system. This is achieved by using four separate smart devices. These devices are, the smart glove, smart shoe, smart cane and the smart wheelchair. These devices logether form the OAM. All these devices would connect to the user's smart-phone using Bluetooth LE. They also connect to the cloud via the smartphone if needed.

3.5.1 Smart Shoe: The smart shoe is meant to help the blind users to commute every day. It can also help blind users who are deaf. The regular shoe is modified to accommodate vibration motors at the front, back, top left and top right corners. The shoe has an inbuilt Bluetooth module to connect with the user's smart-phone. Before travelling the user needs to use Google Maps or any other equivalent application to chart out his travel route. Google Maps can provide turn by turn voice assisted navigation using location services of the smart-phone. The smart shoe uses this route data to help the blind user in travelling. 


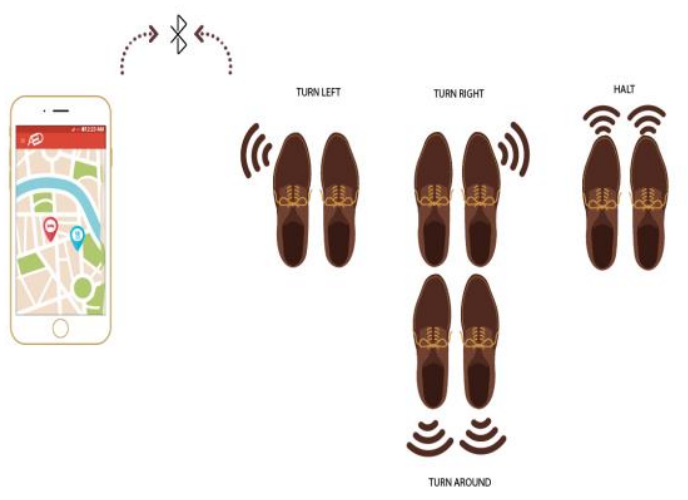

Figure 9. Representation of Navigation using the Smart Shoefor Blind Users. The Shoe is Fitted with Vibration Motors at Various Corner to Signal the Directions for Navigation

When the user approaches a right turn the right shoestarts to vibrate indicating that a right turn has to be made. This experience can be made better if only the top right corner of the right shoe vibrates. Similarly, the top left corner of the left shoe vibrates when the user approaches a left turn. If the user misses any turn and moves ahead both the shoes vibrate at their rear end indicating him to move back, At all times the shoe's are connected to the user's smart-phone yia BluetoothLE. So, even if the user makes a wrong turn by accident Google Maps reroutes, the path to the destination and the updated route is automatically synchronized with the shoe. When the user arrives at his destination both shoes vibrate at the front end to signal the user of his arrival at his destination. This functionality is even more oneficial to the users who are both deaf and blind as they cannot use the voice guided navigation feature of their smart-phone. Thus the smart shoe has high potential to atd the blind in commuting daily with a user friendly mechanism.

3.5.2 Smart Glove: The smart glove is meant to assist blind user to identify objects inside the home and also out-doos accurately. We have already proposed to embed native RFID tags in the various objects inside the house as well as embedding RFID tags in the public landmarks on the streets such as bus stops, ATMs (Automated Teller Machines), footpath barriers etc.
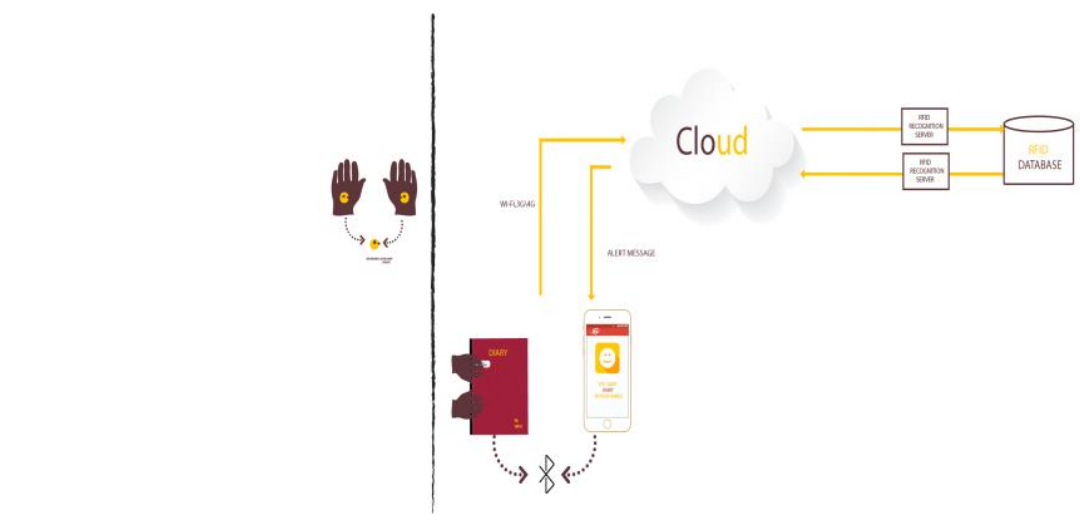

Figure 10. Overview of Functionality of Smart Glove. The Embedded RFID Reader is used to Scan the RFID Tag from a Book. The RFID String is Transmitted to the Cloud where it is Matched Against the RFID Database. The Results are then Delivered to the User's Phone 
The glove will made of comfortable and reliable insulating materials so that they can be used to identify power sockets safely. We propose to embed an RFID reader and Bluetooth module in each of the pair. The smart glove would connect to user's smart phone via Bluetooth LE. When the gloves come in contact with a RFID tagged object it reads the RFID string and transmits it to user's smart-phone. The smart-phone then transmits this RFID string to the cloud where it is then matched against the set of already available tags in the RFID database. The results are then delivered to user's smart-phone. Additional functionalities can also provided using the smart glove. One such functionality would be the ability to control the home devices via gestures.

3.5.3 Smart Wheel-chair: Elderly people, people with physical disabilities and amputees often need a wheel-chair to move from one place to another. A traditional wheel-chair is driven by the user's physical effort and which can be very demanding for these user.

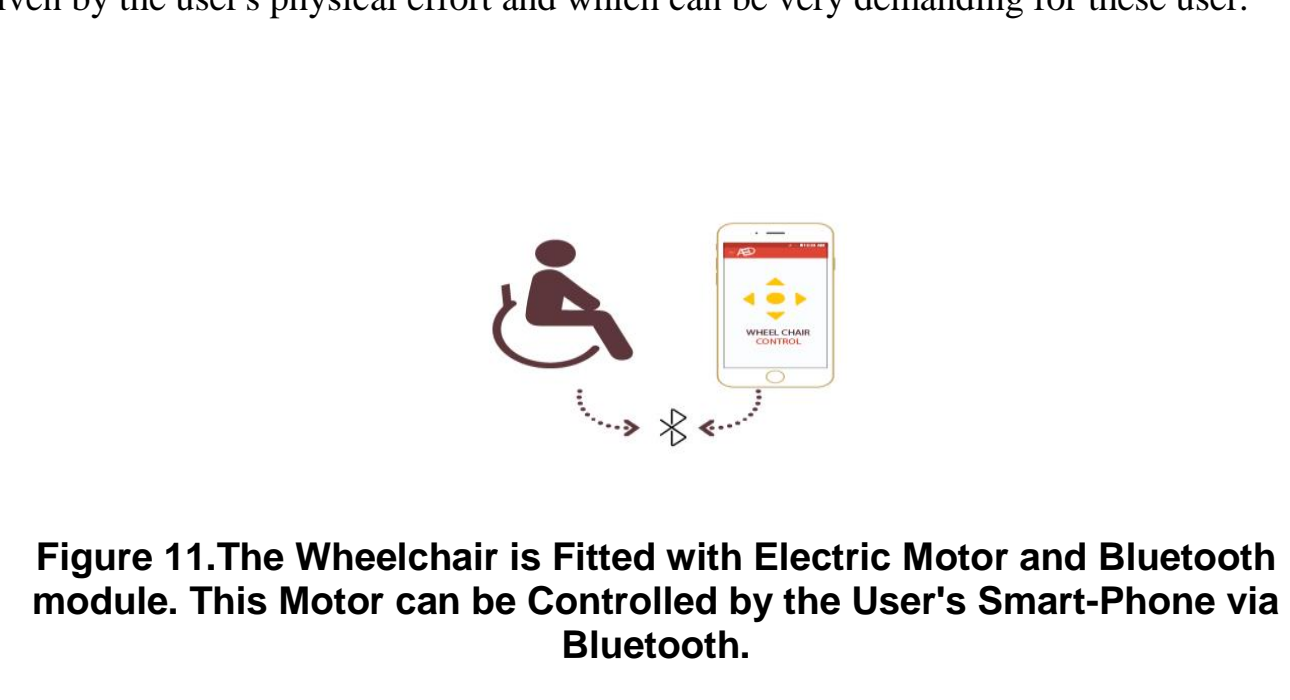

Hence they need someone to assist them to move the wheel-chair. This restricts their ability to move independently. Phis problem can be solved by converting the traditional wheel-chair into a smatt bne by installing a Bluetooth controlled motor. The "smart wheel-chair" would be connected to user's smart phone via Bluetooth LE. This would enable the user to control the movement of wheel chair from his smart-phone, thus removing the need of a helper.

3.5.4 Smart Cane: The Smart cane has been discussed in some related works [1][5]. It serves two purposes which are: object identification and collision avoidance. These will be aseful for blind users. The smart cane is an alternative for the smart glove to identify objects. But due to its dual functionality it is more suited for outdoor use. The cane has embedded RFID reader and ultra-sonic sensor. It is connected to user's smart phone using Bluetooth LE. 


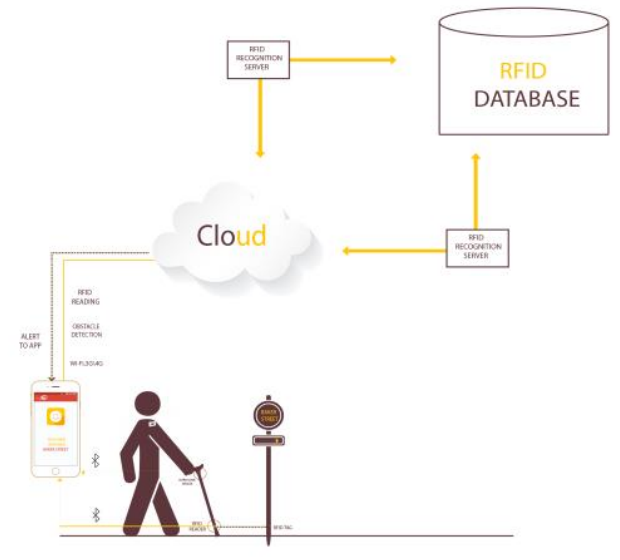

Figure 12.The Smart Cane has Embedded RFID Reader and Ultra-Sonic Sensor. It Communicates to User's Smart-Phone via Bluetooth. The RFID Reader Identifies the Tags Embedded ins de the Landmarks on the Streets. The Ultrasonic Sensor is Directly Connected to User's Smart-Phone. It Provides Low Latency Real Time Collision Avoidance Alerts to the Smart - Phone

The RFID string is matched against the cloud RFII database in the cloud. The data from ultra-sonic can be monitored by the users smart-phone itself or even by server in the cloud. The results will be delivered to user via his smart-phone. The smart cane will be a popular choice because visually impaired users are already accustomed to a normal cane.

\section{Comparison with Existing Systems}

Many systems are already avafitable in the market which focuses on the areas related to health-care, navigation and homeautomation. None of these systems can provide functionality provided by other. Thus an integrated solution which is provided is required. Also it is worth pointing out that currently there exists no standardized platform for implementation of Iob devices. This is a major hindrance in the development of smart systems as they cannot communicate with each other. Brillo and Weave provide an end to end solution to solve this problem.

Home automation systems have been developed by NEST, Samsung and Apple HomeKit.While these platforms provide comprehensive solutions to develop smart homes they do not provide any assistance specific to elderly and disabled. Moreover they do not provide any solution towards health-care and navigation. ALED system combines functionality of these systems in the HAM module by using Weave certified smart devices.

Currently, smart health-care monitoring systems are limited to fitness trackers and discrete high level medical systems. Fitness trackers uses accelerometer and hearth-rate monitor to measure the users fitness metrics. These may not be helpful in providing medical assistance to the elderly and disabled. On the other hand, we have systems that provide specific functionality such as measuring blood-sugar levels in diabetes patients. Accu-chek is a manufacturer which makes a variety of such systems. These provide lack any connectivity to the cloud and hence do not use the concept of IoT. Xiaomi's recently introduced iHealth Box monitors the user's blood pressure level and heart-rate. This device can also synchronize with the user's smart-phone to display history of results. However this device uses its own proprietary OS and cannot be used by non Xiaomi's devices. It is worth mentioning that there are several other devices which can monitor 
ECG (Electro-Cardio Gram), Respiratory rate, body temperature, etc. But a majority of these devices are incapable of sharing their data. They can be turned into smart devices by realizing the concept of IoT which would enable them to communicate with other devices in a network. Moreover, there is a need to combine many of these functionalities into an integrated solution. The ALED system has a module dedicated towards health-care i.e. OBM. The OBM houses the BVM which has a set of smart sensors that monitors the user's health related parameters. This information can be transmitted to relevant medical authorities for online monitoring.

Existing navigation systems such as Google Maps, only provide Voice-Guided turn by turn navigation. There is a need to integrate specially designed hard-ware such as smartshoe proposed in the ALED system to make the navigation even more seamless. For visually impaired the smart cane would be a totally new experience coming from the traditional cane as it helps in object identification and collision avoidance. The smart glove and Bluetooth controlled wheel-chair are also have huge benefits compared to their traditional counter-parts. They help in object identification and greater assistance in movement respectively. Thus the main objective of ALED system is to provide an all in one mobile IoT solution.

\section{Conclusion}

In this paper, we have presented an integrated IoT based solution which should be helpful for the Elderly and Disabled. IoT enbles device to device communication in a network. These devices can range from a simple light bulb to large complex systems such as whole transportation grids. Brillo andWeave are the next generation industry standard for developing IoT devices. The prøposed ALED system uses these standards to connect a variety of devices that would be useful for health-care, navigation, home-automation, etc. The potential benefits of the system have been discussed in all relevant scenarios. The potential applications and challenges have also been discussed. With the discussions in the paper, we anticipate that the proposed ALED system would eventually give rise to a smart and reliable IoT system for assistance of elderly and disabled.

\section{Reference.}

[1]. M. Carmen Domingo, An overview of the Internet of Things for people with disabilities", Journal of Network and Computer Applications, 35, (2012).

[2]. C. F. Pasluosta, 'An emerging Era in the Management of Parkinson's Disease: Wearable Technologies and the Internef of Things", IEEE journal of biomedical and health informatics, vol.19, no. 6, (2015).

[3]. I. F. Akyildiz, M. Pierobon, S. Balasubramaniam, and Y. Koucheryavy,"the internet of bio-nanothings", IEEE Communications Magazine, March (2015).

[4]. E. D'Atri, C. Maria Medaglia, A. Serbanati, Ugo Biader Ceipidor, "A system to aid blind people in the mobility. A usability test and its results", (2016).

[51. S. Chamkamon, P. Tuvaphanthaphiphat, P. Keeratiwintakorn,"A Blind Navigation System Using RFID for Indoor Environments", (2016).

[6]. K. Yelamarthi, D. Haas, D. Nielsen, S. Mothersell, "RFID and GPS Integrated Navigation System for the Visually Impaired", (2016).

[7]. S. Akyol, U. Canzler, "Gesture Control for use in Automobiles", IAPR Workshop on Machine Vision Applications. Nov. 28-30. (2000).

[8]. "Brillo Google Developers", Google Developers, 2016. [Online]. Available: https://developers.google.com/brillo/. (Accessed: 07- May- 2016).

[9]. G. Yang, L. Xie, M. Mantysalo, X. Zhou, Z. Pang, L. Xu, S. Kao-Walter, Q. Chen and L. Zheng, "A Health-IoT Platform Based on the Integration of Intelligent Packaging, Unobtrusive Bio-Sensor, and Intelligent Medicine Box", IEEE Transactions on Industrial Informatics, vol. 10, no. 4, (2014), pp. 2180-2191.

[10]. "Weave Google Developers", Google Developers, 2016. [Online]. Available: https://developers.google.com/weave/. (Accessed: 05- May- 2016).

[11]. "Works with Nest.", Nest, 2016. [Online]. Available: https://nest.com/works-with-nest/. (Accessed: 05May- 2016) 
[12]. YUNCHUAN SUN, HOUBING SONG, ANTONIO J. JARA, RONGFANG BIE," Internet of Things and Big Data Analytics for Smart and Connected Communities", IEEE Access, Special Section on Smart Cities, vol.11, March, (2016).

[13]. Y-Sh Kang, Il-Ha Park, J. Rhee, and Y-H Lee," MongoDB-Based Repository Design for IoT-Generated RFID/Sensor Big Data", IEEE sensors journal, vol. 16, no. 2, (2016).

[14]. A. M. Ortiz, Member, D.Hussein, S. Park, S. N. Han, and Noel Crespi, " The Cluster Between Internet of Things and Social Networks: Review and Research Challenges", IEEE internet of things journal, vol. 1, no. 3, JUNE (2014).

[15]. D. He and S. Zeadally," An Analysis of RFID Authentication Schemes for Internet of Things in Healthcare Environment Using Elliptic Curve Cryptography", IEEE internet of things journal, vol. 2, no. 1, FEBRUARY (2015).

[16]. Y. YIN," The internet of things in healthcare: An overview", Journal of Industrial Information Integration, (2016).

[17]. M. Gentili , R. Sannino , M. Petracca," BlueVoice: Voice communications over Bluetooth Low Energy in the Internet of Things scenario", Elsevier Computer Communications, (2016).

[18]. M. Díaz, C. Martín n, B. Rubio," State-of-the-art, challenges, and open issues in the jntegration of Internet of things and cloud computing", Journal of Network and Computer Applications, vol. 67, (2016), pp. 99-117.

[19]. J. Mineraud, O. Mazhelis, X.Su, S. Tarkoma, "A gap analysis of Internet-of-Things platforms", Elsevier Computer Communications, (2016).

[20]. S-M Seo, S-W Kim, Jin-Woo Jeon, Jee-Hyun Kim, Hee-Soø Kim,Jung-Hwan Cho, Won-Ho Lee, SeHwan Paek, " Food contamination monitoring via intennet of hings, exemplified by using pocket-sized immunosensor as terminal unit", Sensors and Actuators B: Chemical, vol 233, (2016) pp. 148-156,

[21]. H. Jung La," A conceptual framework for trajectory-based medical analytic with IoT contexts", Journal of Computer and System Sciences, vol. 82, no. 4, (2016), pp. 610-626

[22]. C-L Hsu, J. Chuan-Chuan Lin, " An empiricalexamination of consumer adoption of Internet of Things services: Network externalities and concern for information privacy perspectives", Computers in Human Behavior, vol. 62, (2016), pp. 516-527

[23]. S. Park, Y. Kim, H. Chang," An empirical study on-security expert ecosystem in the future IoT service environment", Elsevier Computer \& Electrical Engineering, (2016).

[24]. J. Suarez, J. Quevedo, I. Vidal,D. Corujo, J. Garcia-Reinoso and R. Aguiar, "A secure IoT management architecture based on Information-Centic Aetworking", Journal of Network and Computer Applications, vol. 63, (2016), pp. 190-204.

[25]. M. Razzaque, M. Milojevic Jevric, A. Palade and S. Clarke, "Middleware for Internet of Things: A Survey", IEEE Internet of Things Journal, vol. 3, no. 1, (2016), pp. 70-95

[26]. Al-Fuqaha, M. Gurzani, M. Mohammadi, M. Aledhari and M. Ayyash, "Internet of Things: A Survey on Enabling Technologies, Protocols, and Applications", IEEE Communications Surveys \& Tutorials, vol. 17, no. 4, (2015), pp. 2347-2376

[27]. A. Gill, N. Phennel, D. Lane and V. Phung, "IoT-enabled emergency information supply chain architecture for elderly people. The Australian context", Information Systems, vol. 58, pp. 75-86, 2016.

[28]. Y. Kang, I. Park, L Rhee and Y. Lee, "MongoDB-Based Repository Design for IoT-Generated RFID/Sensor Big Data', IEEE Sensors Journal, vol. 16, no. 2, (2016), pp. 485-497.

[29]. J. Jin, J. Gubbi. S Marusic and M. Palaniswami, "An Information Framework for Creating a Smart City Through Internet of Things", IEEE Internet of Things Journal, vol. 1, no. 2, (2014), pp. 112-121.

[30]. N. Gageik . T. Müller, S. Montenegro, "obstacle detection and collision avoidance using ultrasonic distance sensors for an autonomous quadrocopter", Aerospace Information Technology, University of Würzburg, Germany, September (2012).

[31] "Internet of Things: New Promises for Persons with Disabilities", A G3 ICT Business Case White Paper Series Researched in Cooperation with AT\&T ", July (2015). 